\title{
Clustering of $\mathrm{Y}$ chromosome deletions in subinterval $\mathrm{E}$ of interval 6 supports the existence of an oligozoospermia critical region outside the DAZ gene
}

L Stuppia, V Gatta, G Mastroprimiano, F Pompetti, G Calabrese, P Guanciali Franchi, E Morizio, R Mingarelli, M Nicolai, R Tenaglia, L Improta, V Sforza, S Bisceglia, G Palka

Cattedra di Genetica Umana, Università "G D'Annunzio", Chieti, Italy

L Stuppia

V Gatta

F Pompetti

$G$ Calabrese

P Guanciali Franchi

E Morizio

G Palka

Cattedra di Clinica

Urologica, Università

"G D'Annunzio", Chieti, Italy

G Mastroprimiano

M Nicolai

$\mathbf{R}$ Tenaglia

Ospedale CCS, $S$

Giovanni Rotondo,

Italy

R Mingarelli

Villa del Sole, Napoli, Italy

L Improta

Dipartimento di

Medicina Interna,

Cattedra di

Endocrinologia,

Università "Tor

Vergata", Roma, Italy

V Sforza

S Bisceglia

Correspondence to:

Professor Palka, Via B Buozzi

93, 65100 Pescara, Italy.

Received 14 January 1997 Revised version accepted for publication 28 May 1997

\begin{abstract}
Y chromosome molecular analysis was performed using the STS-PCR technique in 50 patients with oligozoospermia. Microdeletions of interval 6 of the Y chromosome were detected in seven patients, in six of whom subinterval $E$ was affected. All patients retained the RBM1 and DAZ genes, while in one deletion involved the SPGY gene. The size of the deletion was not apparently related to the severity of the disease. These results suggest the presence of an oligozoospermia critical region on the $Y$ chromosome within subinterval $E$ of interval 6.

(F Med Genet 1997;34:881-883)
\end{abstract}

Keywords: oligozoospermia; Y chromosome; PCR; chromosome deletion

Male spermatogenesis involves different genes regulating mitotic and meiotic cell divisions, as well as subsequent differentiation to mature spermatozoa. ${ }^{1}$ Several reports, including consistent observation of microdeletions in infertile patients, have located a critical region on Yq. $^{2-6}$ Based on molecular results, the $Y$ chromosome has been subdivided into seven deletion intervals and the spermatogenesis failure (azoospermia factor, AZF) locus assigned to interval 6 at band Yq1 1. ${ }^{7}$ This latter region has been further subdivided into six subintervals $\left(A, B, C, D, E\right.$, and F).$^{8}$ Three genes have been cloned from interval 6, RBM, ${ }^{9} \mathrm{DAZ},{ }^{10}$ and SPGY, ${ }^{11}$ and are all thought to be related to male infertility. The existence of additional spermatogenesis genes within this interval cannot be excluded. In fact, deletion in interval 6 produces distinct phenotypes of the number and stage of germ cells in testis tissue sections. Most tubules had a complete absence of germ cells. In some tubules, germ cells at different developmental stages (spermatogonia, spermatocytes, spermatids) were recognised. Some men were diagnosed as having oligozoospermia. In addition, some infertile men show microdeletions outside the DAZ gene. ${ }^{5}$ Vogt et $a l^{11}$ have argued that no fewer than three AZF loci exist, $\mathrm{AZFa}, \mathrm{AZFb}$, and $\mathrm{AZFc}$, the latter including the DAZ and SPGY genes. AZFa and $\mathrm{AZFb}$ deletions result in azoospermia, while AZFc deletions at subintervals D, E, and $F$ can cause both azoospermia and oligozoospermia. We have reported an oligozoospermic patient and his father, both with a del(Yq11), where molecular analysis disclosed maintenance of the subinterval $\mathrm{E}$ in the father and its deletion in the son. ${ }^{12}$ This has suggested to us a relationship between subinterval $\mathrm{E}$ deletion and oligozoospermia. ${ }^{12}$ In order to test this hypothesis, a systematic molecular analysis using STS-PCR ${ }^{8}$ was performed in 50 patients with idiopathic oligozoospermia.

\section{Materials and methods} PATIENTS

Fifty consecutive subjects with idiopathic oligozoospermia, including a previously reported case, ${ }^{12}$ with a mean age of 34 years (range 23-48) were entered into this study. The diagnosis was made on the basis of semen analysis according to the WHO guidelines. ${ }^{13}$ All subjects had a sperm count below 20 million per ml ejaculate, with or without additional abnormalities of sperm motility or head and tail morphology. Each patient was carefully examined to rule out other causes of infertility. FSH, LH, and TSH values were available for all patients. A normal male karyotype was detected in 50 metaphases from each patient except one, who showed a del(Yq11), as previously reported. ${ }^{12}$

\section{STS-PCR ANALYSIS}

Each patient was examined for 27 loci mapped to interval 6 of the $\mathrm{Y}$ chromosome using the STS-PCR approach. These loci represent a selection of STS primers from subintervals A-F. In detail, we analysed sY129, sY130, sY131, sY132, sY134, and sY164 (subinterval A); sY138, sY143, and MK5 (subinterval B); sY139, sY153, sY150, sY152, and sY220 
Table 1 Clinical, cytogenetic, and molecular data of the patients with $Y$ chromosome microdeletions

\begin{tabular}{|c|c|c|c|c|c|c|c|}
\hline Patient & Age & Sperm count & $F S H$ & $L H$ & $T S H$ & Karyotype & Deleted STSs \\
\hline 1 & 39 & $<2 \mathrm{M} / \mathrm{ml}$ & $9 \mathrm{mIU} / \mathrm{ml}(\mathrm{NR}<15)$ & $4.2 \mathrm{mIU} / \mathrm{ml}(\mathrm{NR}<20)$ & $7 \mathrm{ng} / \mathrm{ml}$ (NR 3-10) & $46, X Y$ & sY273, sY269 \\
\hline 2 & 26 & $5 \mathrm{M} / \mathrm{ml}$ & $12 \mathrm{mIU} / \mathrm{ml}(\mathrm{NR}<15)$ & $3 \mathrm{mIU} / \mathrm{ml}(\mathrm{NR}<20)$ & $5 \mathrm{ng} / \mathrm{ml}(\mathrm{NR} 3-10)$ & $46, \mathrm{XY}$ & $\begin{array}{l}\text { SPGY, sY272, sY273, sY269, } \\
\text { sY243 }\end{array}$ \\
\hline 3 & 23 & $<2 \mathrm{M} / \mathrm{ml}$ & $8 \mathrm{mIU} / \mathrm{ml}(\mathrm{NR}$ 1-14) & $\begin{array}{l}2.6 \mathrm{mIU} / \mathrm{ml}(\mathrm{NR} \\
1.5-9.2)\end{array}$ & $4 \mathrm{ng} / \mathrm{ml}$ (NR 3-10) & $46, X Y$ & sY147 \\
\hline 4 & 28 & $7 \mathrm{M} / \mathrm{ml}$ & $16 \mathrm{mIU} / \mathrm{ml}(\mathrm{NR}<15)$ & $6.3 \mathrm{mIU} / \mathrm{ml}(\mathrm{NR}<20)$ & $5 \mathrm{ng} / \mathrm{ml}$ (NR 3-10) & $46, X Y$ & sY269, sY243, sY167 \\
\hline 5 & 32 & $<2 \mathrm{M} / \mathrm{ml}$ & $5.4 \mathrm{mIU} / \mathrm{ml}$ (NR 1-9) & $3.22 \mathrm{mIU} / \mathrm{ml}(\mathrm{NR} 1-5)$ & $5 \mathrm{ng} / \mathrm{ml}(\mathrm{NR} 3-10)$ & $46, X, \operatorname{del}(Y)(q 11)$ & $\begin{array}{l}\text { sY269, sY243, sY167, sY166, } \\
\text { sY158 }\end{array}$ \\
\hline 6 & 30 & $9 \mathrm{M} / \mathrm{ml}$ & $\mathrm{mIU} / \mathrm{ml}(\mathrm{NR}<15)$ & $3.8 \mathrm{mIU} / \mathrm{ml}(\mathrm{NR}<20)$ & $7 \mathrm{ng} / \mathrm{ml}$ (NR 3-10) & $46, X Y$ & sY272, sY273 \\
\hline 7 & 27 & $8 \mathrm{M} / \mathrm{ml}$ & $45 \mathrm{mIU} / \mathrm{ml}(\mathrm{NR} 2-20)$ & $17 \mathrm{mIU} / \mathrm{ml}$ (NR 2-10) & $6 \mathrm{ng} / \mathrm{ml}(\mathrm{NR} 3-10)$ & $46, X Y$ & sY147, sY273 \\
\hline
\end{tabular}

(subinterval C); sY155, sY147, sY149, sY254, sY255, and SPGY1 (subinterval D); sY272, sY273, sY269, and sY243 (subinterval E); and sY167, sY158, and sY166 (subinterval F). MK5, sY254-255, and SPGY1 are specific for the genes RBM1, DAZ, and SPGY, respectively. Amplifications were performed with 30 cycles of $94^{\circ} \mathrm{C}$ for one minute, $61^{\circ} \mathrm{C}$ for one minute, and $72^{\circ} \mathrm{C}$ for one minute. PCR products were analysed on a $2 \%$ agarose gel or on a $6 \%$ acrylamide gel, after staining with ethidium bromide. An STS was considered as absent only after at least three amplification failures, in the presence of a successful amplification of an internal control. To exclude the presence of genetic polymorphisms, STS-PCR analysis was also performed in the fathers of three patients (patients 3, 4, and 5) and in 10 fertile subjects, which were used as positive controls.

\section{Results}

PCR amplifications produced a band of the expected size for all the 27 loci investigated in the controls and in 43 patients. Seven patients (14\%) showed a deletion of one or more STSs, namely sY273 and sY269 (patient 1), sY272, sY273, sY269, sY243, and SPGY1 (patient 2), sY147 (patient 3), sY269, sY243, and sY167 (patient 4), sY269, sY243, sY167, sY158, and sY166 (patient 5), sY272 and sY273 (patient 6), and sY147 and sY272 (patient 7). All deletions were interstitial, except in one patient (patient 5) where it was terminal. PCR amplification was also performed on the fathers of three of these seven patients (patients 3, 4, and 5). The fathers of two of these (patients 3 and 4) did not show microdeletions, while the father of patient 5 had a deletion of sY167, sY158, and sY166. The fathers of the other four patients with microdeletions (patients 1,2, 6 , and 7) were not available. A summary of data of the seven patients with microdeletions is shown in table 1 , and a deletion map is shown in fig 1 .

\section{Discussion}

Idiopathic oligozoospermia is the most common cause of male infertility, affecting 3-4\% of men. ${ }^{14}$ Unlike azoospermic patients, subjects with oligozoospermia rarely show chromosome abnormalities. In recent years, several studies have reported microdeletions of interval 6 of the $\mathrm{Y}$ chromosome in both azoospermic and oligozoospermic patients. ${ }^{3-6}$ In particular, Reijo et $a l,{ }^{15}$ using STS-PCR, described two subjects with microdeletions of interval 6 , involving

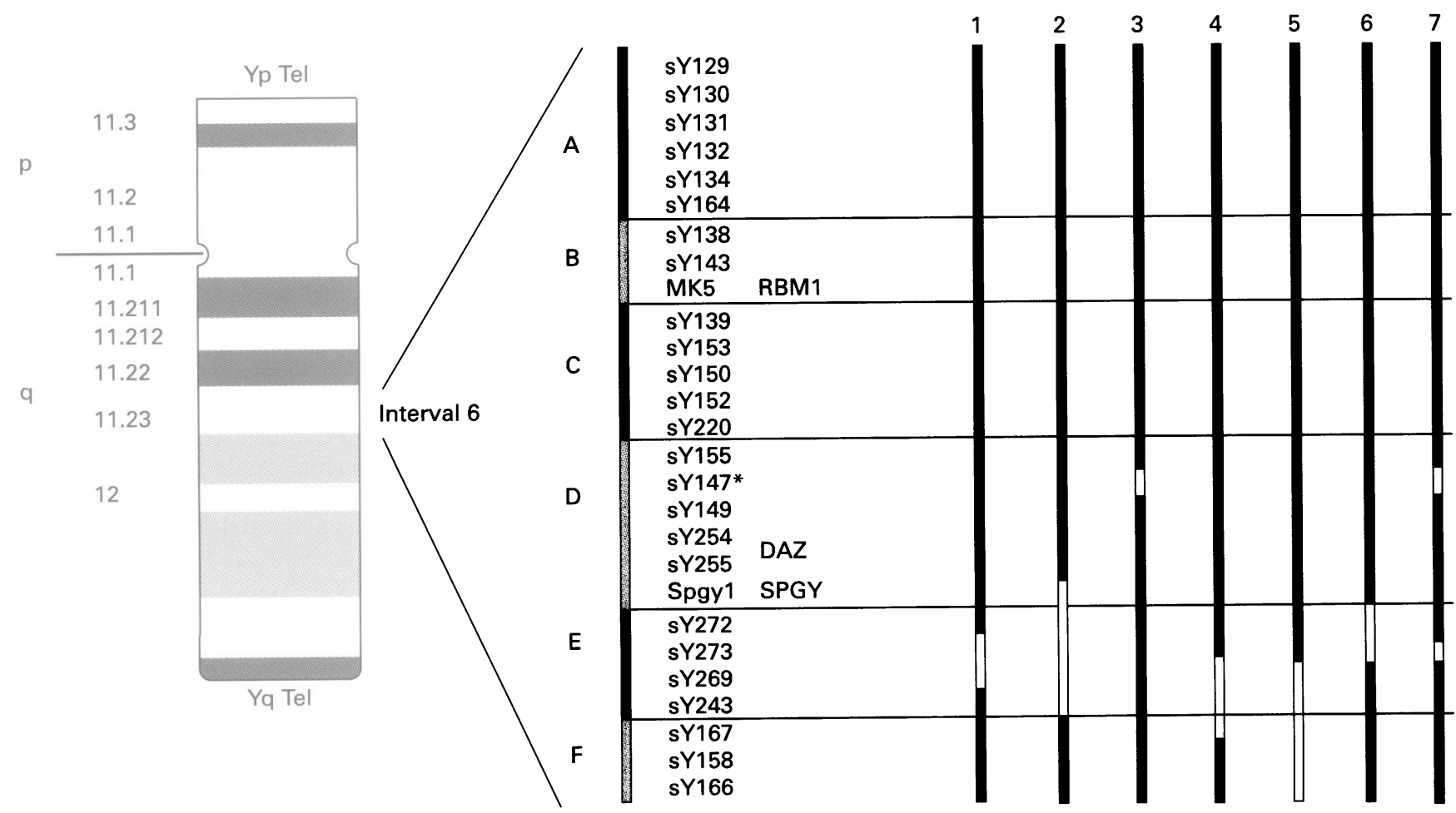

Figure 1 Diagram showing microdeletions of interval 6 in seven patients with oligozoospermia. Filled bars=presence of the STS, empty bars=absence of the $S T S, *_{S} Y 147$ has also been mapped in subinterval $E$. 
subintervals $C, D, E$, and $F$. The deletion of the DAZ gene in both cases supported a pathogenetic role of this gene in oligozoospermia as well. Vogt et al, ${ }^{11}$ using the same approach, found additional oligozoospermia patients with microdeletions in the distal interval $6, \mathrm{AZFc}$, a region of at least $500 \mathrm{~kb}$, encompassing subintervals $\mathrm{D}, \mathrm{E}$, and $\mathrm{F}$ and including the DAZ and SPGY genes. We have argued that the oligozoospermia critical region is limited to subinterval $\mathrm{E}$, outside the DAZ gene, based on the observation of an oligozoospermic patient with a microdeletion in this subinterval. ${ }^{12}$ In the present study, we found microdeletions of subinterval $E$ in six of 50 patients with oligozoospermia. The seventh patient was deleted for $\mathrm{sY} 147$, whose map position is still debated between subintervals $\mathrm{D}$ and $\mathrm{E} .{ }^{816}$ The discovery of a second patient with deleted sY147, associated with loss of sY272 which maps within interval E, supports physical mapping of sY147 to the proximal subinterval E. All patients retained the DAZ and RBM1 genes and only one had a deletion of SPGY. These data confirm our previous claim about the presence of an oligozoospermia critical region within subinterval $E^{12}$ and further narrow this critical region. The study and characterisation of this region and the identification of one or more genes within subinterval E will improve current understanding of the biological basis of oligozoospermia. Our results indicate that $14 \%$ of oligozoospermic patients have $\mathrm{Y}$ chromosome de novo microdeletions which are probably related to the disease. At present no evidence has been found for a different genotype-phenotype correlation. We conclude that STS-PCR is valuable for screening the microdeletions in interval 6 of the $Y$ chromosome in patients with infertility of unknown origin.
1 Wolgemuth DJ, Rhee K, Wu S, Ravnik SE. Genetic control of mitosis, meiosis and cellular differentiation during mammalian spermatogenesis. Reprod Fertil Dev 1995;7:669-83.

$2 \mathrm{Ma} \mathrm{K}$, Sharkery A, Kirsch S, et al. Towards the molecular localisation of the AZF locus: mapping of microdeletions in azoospermic man within 14 subintervals of interval 6 of the Y chromosome. Hum Mol Genet 1992;1:29-33.

3 Nagafuchi S, Namiki M, Nakahori Y, Kondoh N, Okuyama A, Nakagome Y. A minute deletion of the $Y$ chromosome in men with azoospermia. F Urol 1993;150:1155-7.

4 Stuppia L, Mastroprimiano G, Calabrese G, Peila R, Tenaglia R, Palka G. Microdeletions in interval 6 of the Y chromosome detected by STS-PCR in 6 of 33 patients with idiopathic oligo- or azoospermia. Cytogenet Cell Genet 1996;72: 155-8.

5 Najmabadi H, Huang V, Yen P, et al. Substantial prevalence of microdeletions of the Y-chromosome in infertile men with idiopathic azoospermia and oligozoospermia detected using a sequence-tagged site-based mapping strategy. $f$ Clin using a sequence-tagged site-based map
Endocrinol Metab 1996;81:1347-52.

6 Kobayashi K, Mizuno K, Hida A, et al. PCR analysis of the $\mathrm{Y}$ chromosome long arm in azoospermic patients: evidence for a second locus required for spermatogenesis. Hum Mol Genet 1994;3:1965-7.

7 Vergnaud G, Page DC, Simmler MC, et al. A deletion map of the human $\mathrm{Y}$ chromosome based on DNA hybridization. Am $\mathcal{~}$ Hum Genet 1986;38:109-24.

8 Vollrath D, Foote S, Hilton A, et al. The human $\mathrm{Y}$ chromosome: a 43-interval map based on naturally occurchromosome: a 43-interval map base

$9 \mathrm{Ma} \mathrm{K}$, Inglis JD, Sharkey A, et al. A Y chromosome gene family with RNA-binding protein homology: candidates for the azoospermia factor AZF controlling human spermatogenesis. Cell 1993;75:1287-95.

10 Reijo R, Lee TY, Salo P, et al. Diverse spermatogenic defects in humans caused by $\mathrm{Y}$ chromosome deletions encompassing a novel RNA-binding protein gene. Nat Genet 1995;10: 383-93.

11 Vogt PH, Edelmann A, Kirsh S, et al. Human Y chromosome azoospermia factors (AZF) mapped to different subregions in Yq11. Hum Mol Genet 1996;5:933-43.

12 Stuppia L, Calabrese G, Guanciali Franchi P, et al. Widening of a Y chromosome interval 6 deletion transmitted from father to his infertile son accounts for an oligozoospermia critical region distal to the RBM1 and DAZ genes. $\mathrm{Am} F$ Hum Genet 1996;59:1393-4.

13 World Health Organization. WHO laboratory manual for the examination of human semen and $S$-cervical mucus interaction. 3rd ed. Cambridge: Cambridge University Press, 1992.

14 Hull MGR, Glazener CMA, Kelly NJ, et al. Population study of causes, treatment and outcome of infertility. $B M F$ 1985;291:1693-7.

15 Reijo R, Alagappan RK, Patrizio P, Page DC. Severe oligozoospermia resulting from deletions of azoospermia factor gene on Y chromosome. Lancet 1996;347:1290-1.

16 Foote S, Vollrath D, Hilton A, Page DC. The human Y chromosome: overlapping DNA clones spanning the euchromatic regions. Science 1992;258:60-6. 